\title{
Sensitivity of Transfer Capability Margins With a Fast Formula
}

\author{
Scott Greene, Member, IEEE, Ian Dobson, Senior Member, IEEE, and Fernando L. Alvarado, Fellow, IEEE
}

\begin{abstract}
Bulk power transfers in electric power systems are limited by transmission network security. Transfer capability measures the maximum power transfer permissible under certain assumptions. Once a transfer capability has been computed for one set of assumptions, it is useful to quickly estimate the effect on the transfer capability of modifying those assumptions. This paper presents a computationally efficient formula for the first order sensitivity of the transfer capability with respect to the variation of any parameters. The sensitivity formula is very fast to evaluate. The approach is consistent with the current industrial practice of using dc load flow models and significantly generalizes that practice to more detailed ac power system models that include voltage and reactive power limits. The computation is illustrated and tested on a 3357 bus power system.
\end{abstract}

Index Terms-Optimization, power system control, power system security, power transmission planning, sensitivity.

\section{INTRODUCTION}

$\mathbf{T}$ RANSFER capability indicates how much a particular bulk power transfer can be changed without compromising system security under a specific set of assumptions. The increased attention to the economic value of transfers motivates more accurate and defensible transfer capability computations.

A variety of applications in both planning and operations require the repetitive computation of transfer capabilities. Transfer capabilities must be quickly computed for various assumptions representing possible future system conditions and then recomputed as system conditions change. The usefulness of each computed transfer capability is enhanced if the sensitivity of the transfer capability is also computed [10], [15]. This paper shows how to quickly compute these sensitivities in a general and efficient way. The sensitivities can be used to estimate the effect on the transfer capability of variation in simultaneous transfers, assumed data, and system controls. A web site [6] is available to calculate these sensitivities on sample power systems and further illustrate their use.

While there is general agreement on the overall purpose and outline of transfer capability determination, the precise requirements for such computations vary by region and are evolving. In this paper we focus on the fast computation of the sensitivity of the transfer margin, not the computation of the transfer margin itself. However, to explain the sensitivity computation we need

\footnotetext{
Manuscript received January 4, 2000; revised July 13, 2001. This work was supported in part by NSF Research Centers Small Firms Collaborative R\&D Initiative Grant EEC-9815325.

S. Greene is with L. R. Christensen Associates, Madison, WI 53705 USA.

I. Dobson and F. L. Alvarado are with the Power Systems Engineering Research Center, ECE Department, University of Wisconsin, Madison, WI 53706 USA (e-mail: dobson@engr.wisc.edu).

Publisher Item Identifier S 0885-8950(02)00891-X.
}

to first discuss a generic transfer margin computation. The sensitivity computation is largely independent of the method used to obtain the transfer margin.

\section{GENERIC TRANSFER MARgin COMPUTATION}

We assume that an initial transfer margin computation has established the following.

1) A secure, solved base case consistent with the study operating horizon.

2) Specification of transfer direction including source, sink, and loss assumptions.

3) A solved transfer-limited case and a binding security limit. The binding security limit can be a limit on line flow, voltage magnitude, voltage collapse or other operating constraint. Further transfer in the specified direction would cause the violation of the binding limit and compromise system security.

4) The transfer margin is the difference between the transfer at the base case and the limiting case.

Calculations of available transfer capability (ATC), capacity benefit margin (CBM), and transfer reliability margin (TRM) typically require that this generic transfer margin computation be repeated for multiple combinations of transfer directions, base case conditions, and contingencies [13], [15].

The generic transfer margin computation can be implemented with a range of power system models and computational techniques. One convenient and standard practice is to use a dc power flow model to establish transfer capability limited by line flow limits. The limiting cases are then checked with further ac load flow analysis to detect possibly more limiting voltage constraints.

Alternatively, a detailed ac power system model can be used throughout and the transfer margin determined by successive ac load flow calculations [10] or continuation methods [1]-[3], [16]. A related approach [e.g., Electric Power Research Institute's TRACE] uses an optimal power flow where the optimization adjusts controls such as tap and switching variables to maximize the specified transfer subject to the power flow equilibrium and limit constraints. The formulations in [10] and [18] show the close connection between optimization and continuation or successive load flow computation for transfer capability determination. The sensitivity methods of this paper are applicable to transfer margins computed by optimization, continuation or other methods. The implementation of the sensitivity formula can take advantage of numerical byproducts of common sequential linear programming (LP) techniques. 
Methods based on ac power system models are slower than methods using dc load flow models but do allow for consideration of additional system limits and more accurate accounting of the operation guides and control actions that accompany the increasing transfers. Under highly stressed conditions the effects of tap changing, capacitor switching, and generator reactive power limits become significant. A combination of $\mathrm{dc}$ and ac methods may be needed to achieve the correct tradeoff between speed and accuracy. The methods in this paper account directly for any limits which can be deduced from equilibrium equations such as dc or ac load flow equations or enhanced ac equilibrium models.

\section{SENSITIVITy COMPUTATION}

\section{A. System Modeling}

Assume a general power system equilibrium model written as $n$ equations

$$
0=f(x, \lambda, p)
$$

where

$x \quad n$ dimensional state vector that includes voltage magnitudes, angles, branch flows, and generator MW and MVAR outputs;

$\lambda \quad$ vector of generator MW output set points and/or scheduled net area exports;

$p$ parameter vector including regulated voltage set points, generator load sharing factors, load and load model parameters and tap settings.

The limits on line flows, voltage magnitudes, or generator reactive power outputs are modeled by inequalities in the states

$$
x_{i}^{\min } \leq x_{i} \leq x_{i}^{\max } \quad i=1, \ldots, n .
$$

Due to the modeling of operator actions and generator limits, the equilibrium equations and the physical quantities represented by the $x$ and $p$ vectors can change under varying conditions and transfer levels. For example, when a nonslack generator is operated within its reactive power limits, the reactive power output and angle at the generator bus are components of $x$ and the regulated bus voltage and real power output are components of $p$. However, when the same generator is at a reactive power limit, the generator bus voltage and angle are components of $x$ and the real and reactive power output are components of $p$.

Base Case: The base case specifies the nominal value $\lambda_{0}$ of the generator outputs and net area exports.

Transfer Specification: The transfer is specified by changes to the vector $\lambda$. The transfer direction describes how $\lambda$ changes as the transfer increases so that

$$
\lambda=\lambda_{0}+k t
$$

where $t$ is the transfer amount and $k$ is a unit vector describing the transfer direction. For the simple case of net exports increasing from one area matched by reduction in net export from another area, the transfer direction $k$ is a column vector with 1 in the row corresponding to the source area export equation and -1 in the row corresponding to the sink area export equation. For transfers specified by changes in individual bus injections, $k$ is a column vector with positive entries at the source buses and negative entries at the sink buses.

Transfer-Limited Case: Identification of a solved transferlimited case yields an equilibrium solution $\left(x_{*}, \lambda_{*}, p_{*}\right)$ and an additional constraint referred to as the binding limit. The equilibrium equations that model the power system at the binding limit are written

$$
0=F(x, \lambda, p)
$$

When a limit is encountered, one of the limit equations $x_{i}=$ $x_{i}^{\min }$ or $x_{i}=x_{i}^{\max }$ holds for some $i$. We write the applicable equation for the binding limit in the general form

$$
0=E(x, \lambda, p) \text {. }
$$

The form (2) also encompasses more general limits. At the binding limit

$$
\begin{aligned}
& F\left(x_{*}, \lambda_{*}, p_{*}\right)=0 \\
& E\left(x_{*}, \lambda_{*}, p_{*}\right)=0 .
\end{aligned}
$$

Transfer Margin: The transfer margin is the change in the transfer between the base case and the transfer-limited case. Since $\lambda_{*}=\lambda_{0}+k T$, the transfer margin is $T$.

\section{B. Sensitivity Formula}

Once the binding limit and the corresponding transfer-limited solved case have been found, the sensitivity of the transfer margin $T$ can be evaluated. The sensitivity of $T$ to the parameter $p$, often written as $\partial T / \partial p$ and here written as $T_{p}$, is computed using a formula derived in Appendices A and B

$$
T_{p}=\frac{-\left.w\left(\begin{array}{l}
F_{p} \\
E_{p}
\end{array}\right)\right|_{\left(x_{*}, \lambda_{*}, p_{*}\right)}}{\left.w\left(\begin{array}{l}
F_{\lambda} k \\
E_{\lambda} k
\end{array}\right)\right|_{\left(x_{*}, \lambda_{*}, p_{*}\right)}}
$$

where

$F_{p}$ and $E_{p} \quad$ derivatives of the equilibrium and limit equations with respect to $p$;

$F_{\lambda} k$ and $E_{\lambda} k$ derivatives of the equilibrium and limit equations with respect to the amount of transfer $t$;

$w$ nonzero row vector orthogonal to the range of the Jacobian matrix $J$ of the equilibrium and limit equations;

where

$$
J=\left.\left(\begin{array}{l}
F_{x} \\
E_{x}
\end{array}\right)\right|_{\left(x_{*}, \lambda_{*}, p_{*}\right)} \cdot
$$

The row vector $w$ is found by solving the linear system

$$
w J=0 \text {. }
$$

Since $J$ has one more row than column, there is always a nonzero vector $w$ that solves (4). $J$ generically has full column rank, so that $w$ is unique up to a scalar multiple. The sensitivity $T_{p}$ computed from (3) is independent of the scalar multiple. 
The first order estimate of the change in transfer margin corresponding to the change in $p$ of $\Delta_{p}$ is

$$
\Delta T=T_{p} \Delta p
$$

If the binding limit is an immediate voltage collapse due to a reactive power limit [5], then the analysis of this paper applies with (2) becoming $Q_{i}=Q_{i}^{\max }$. If the binding limit is voltage collapse due to a fold bifurcation, the sensitivity formula of [8] applies.

\section{Computational Efficiency}

Once the transfer-limited solution is obtained, the margin estimates corresponding to varying a large number of different parameters can be obtained for little more computational effort than solving the sparse linear equations (4) for $w$. Solving (4) is roughly equivalent to one Newton iteration of a load flow solution. Note that $w$ need only be computed once but can be used to find the sensitivity with respect to any number of parameters. If a sequential LP is used to determine the transfer margin as part of an optimization program, then $w$ is found from the Lagrange multipliers obtained at the last LP solution. The remaining computations (3) and (5) needed for the estimates require only sparse matrix-vector multiplications.

The Jacobian matrix $J$ in (4) is available, often in factored form, from the computation of the transfer-limited solution by Newton based methods. The matrix $F_{p}$ in (3) is different for each parameter $p$ but its construction is a simple sparse index operation, especially when the parameters appear linearly.

The sensitivity of the transfer capability with respect to thousands of changes in load, generation, interarea transfers, or voltage set points can be obtained in less time than a single ac load flow solution.

\section{3357 BUS EXAMPLE}

The application of sensitivity formula (3) is illustrated using a 3357 bus model of a portion of the North American eastern interconnect. The model contains a detailed representation of the network operated by the New York independent system operator and an equivalent representation of more distant portions of the network. From a base case representative of a severely stressed power system, small increases in transfer between Ontario Hydro and New York City lead to low voltages, cascading generator reactive power limits, and finally voltage instability. The sensitivity formulas are used to identify effective control action to avoid low voltage and reactive power limit conditions, and to estimate the effects of variation in transfers and loading on the security of the system.

Base Case: The base case is motivated by a scenario identified as problematic in the New York Power Pool summer 1999 operating study. The loss of two $345 \mathrm{KV}$ lines, Kintigh-Rochester and Rochester-Pannell Road during high west to east transfer leads to low voltage conditions at the Rochester $345 \mathrm{kV}$ bus. At the base case solution, the voltage at the Rochester $345 \mathrm{kV}$ bus is $333 \mathrm{kV}$, slightly above the $328 \mathrm{kV}$ low voltage rating.
TABLE I

Net Zone Exports in MW at Base CASE. THE INITIAL Voltage Limit AT THE ROCHESTER 345 KV BUS, AND THE FinAL REACTIVE POWER LIMIT AT DANKSAMMER

\begin{tabular}{cccc} 
ZONE & $\begin{array}{c}\text { net export } \\
\text { base case }\end{array}$ & $\begin{array}{c}\text { net export } \\
\text { voltage limit }\end{array}$ & $\begin{array}{c}\text { net export } \\
\text { VAR limit }\end{array}$ \\
\hline NYC & -4806 & -4906 & -5046 \\
OH & 4080 & 4180 & 4320 \\
HQ & 976 & 976 & 976 \\
PJM & -3422 & -3422 & -3422 \\
ISO-NE & -28 & -28 & -28
\end{tabular}

Limiting Events: From the base case, a sequence of ac load flow solutions are obtained for increasing levels of export from Ontario Hydro and increasing demand in the New York City zone. A $100 \mathrm{MW}$ increase in this transfer results in the voltage at the $345 \mathrm{kV}$ Rochester bus reaching its low voltage rating of $328 \mathrm{kV}$. Additional transfer leads to several low voltages and nine additional generating units reaching maximum reactive power limits. Finally, for transfer of $140 \mathrm{MW}$ beyond that corresponding to the Rochester voltage limit, a reactive power limit at one of the Danksammer generating units leads to immediate voltage instability [5]. (System behavior under the stressed conditions is unstable without voltage regulation at Danksammer.)

Table I shows the net exports for five of the zones at the base case and at two different limits. The transfer margin to the voltage limit is $100 \mathrm{MW}$ and the transfer margin to the critical reactive power limit is $240 \mathrm{MW}$. Since it is of interest how avoiding the low voltage limit also improves the margin to voltage instability, we compute the sensitivities of both these margins.

\section{A. Sensitivity to Regulated Voltage Set Points}

The sensitivity of the transfer margins to the Rochester voltage limit and the Danksammer reactive power limit with respect to all parameters is obtained using (3). Ranking of all the NY ISO generator buses according to the sensitivity of the transfer margins with respect to regulated generator voltages indicates that the regulated voltage with the greatest effect on the transfer margin to the Rochester voltage limit and the second greatest effect on the margin to the Danksammer reactive power limit is the Hydro facility in Niagara.

Fig. 1 shows the linear estimate for the change in transfer margin to the voltage limit as a function of the voltage set point at the Niagara generator. The estimates are compared with actual values computed by ac loadflow analysis represented by the circles in Fig. 1. The actual values are obtained by incrementing the voltage set point and rerunning the transfer capability calculation. In effect, the incremental variation method of [9] is used to check the sensitivity formula. Fig. 2 compares the linear estimate with actual values computed by ac loadflow analysis for the change in the transfer margin to the Danksammer reactive power limit as a function of the Niagara voltage set point. Figs. 1 and 2 show that the estimates are accurate for $a \pm 5 \%$ variation in the regulated output voltage of the Niagara unit. Note that for both limits, setting the voltage set point greater than 1.07 p.u. does not improve the margin as predicted because at that voltage the generator reactive power output reaches its maximum before the transfer limit is encountered. 


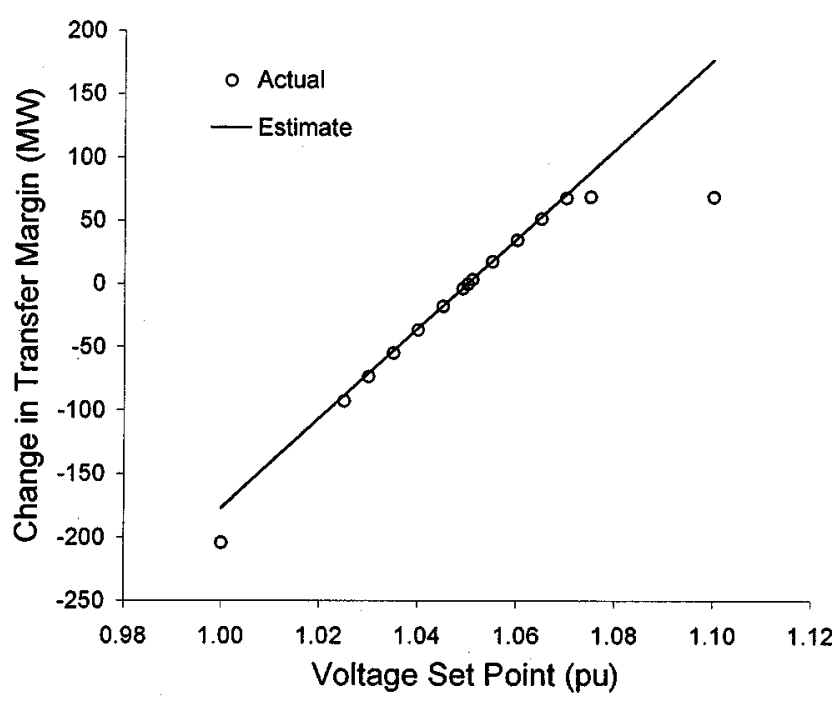

Fig. 1. Effect of regulated output voltage on margin to voltage limit.

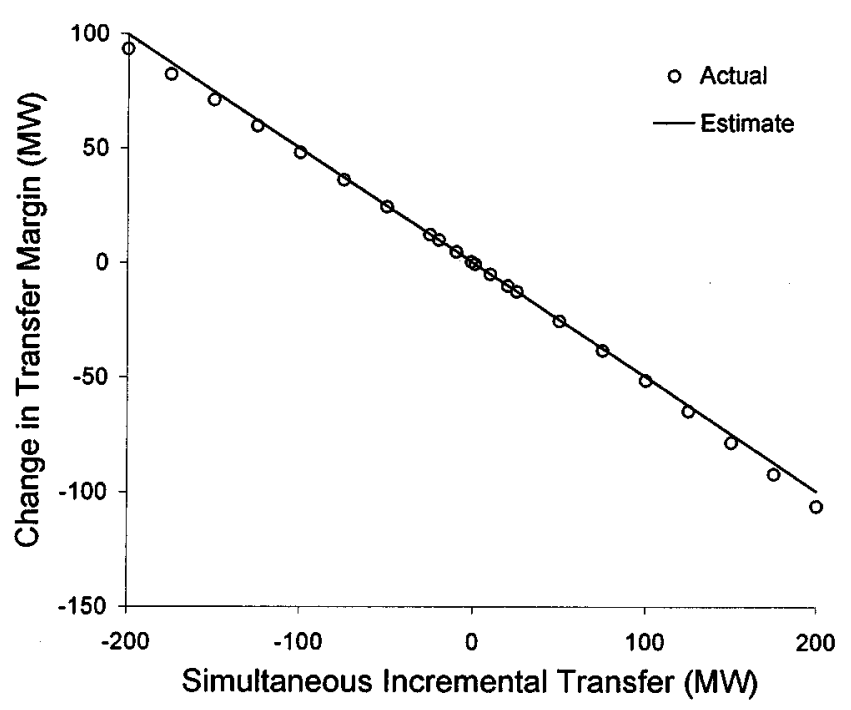

Fig. 2. Effect of regulated output voltage on margin to critical VAR limit.

\section{B. Sensitivity to Simultaneous Transfers}

One concern is the effect of simultaneous transfers on the computed transfer margins. Figs. 3 and 4 show the effects on the voltage and reactive power limited transfer margins of a simultaneous Hydro Quebec to PJM transfer. The simultaneous transfer affects the reactive power limit more than the voltage limit, and the sensitivity based estimates are accurate for $\mathrm{a} \pm 200$ MW transfer variation, which is a $20 \%$ variation in export from Hydro Quebec.

\section{Sensitivity to Load Variation}

Another concern is load forecast error. For example, consider the effect of load variation in the Albany region on the transfer margins. The real and reactive power loads in Albany are changed keeping constant power factor. The estimates are compared with the actual values computed directly from ac loadflow analysis in Figs. 5 and 6. The results are very accurate for $\pm 200 \mathrm{MW}$ total load variation, but less accurate for \pm 400 MW. The base case Albany zone load is 2000 MW.

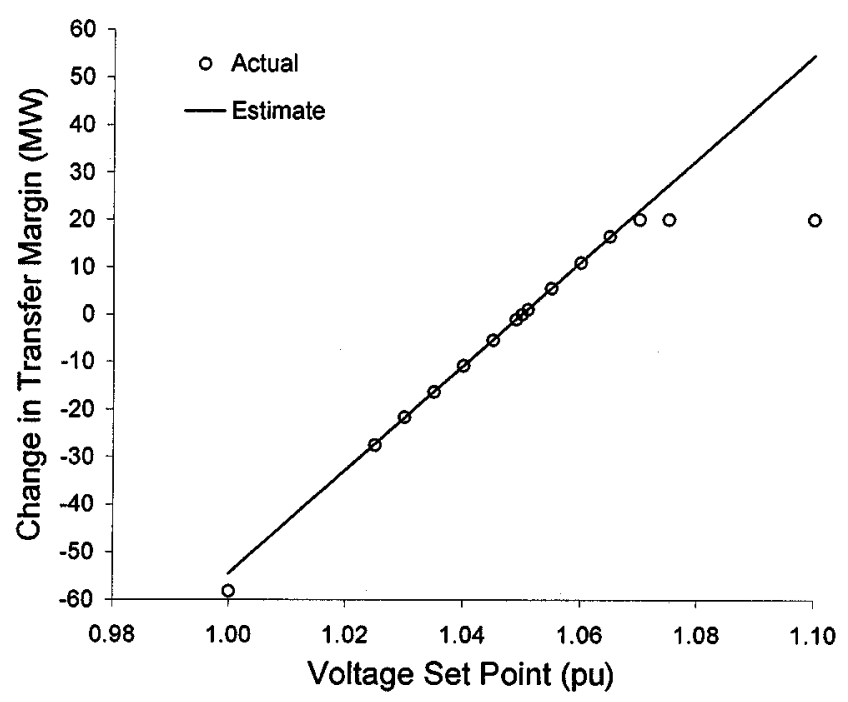

Fig. 3. Effect of simultaneous transfer on margin to voltage limit.

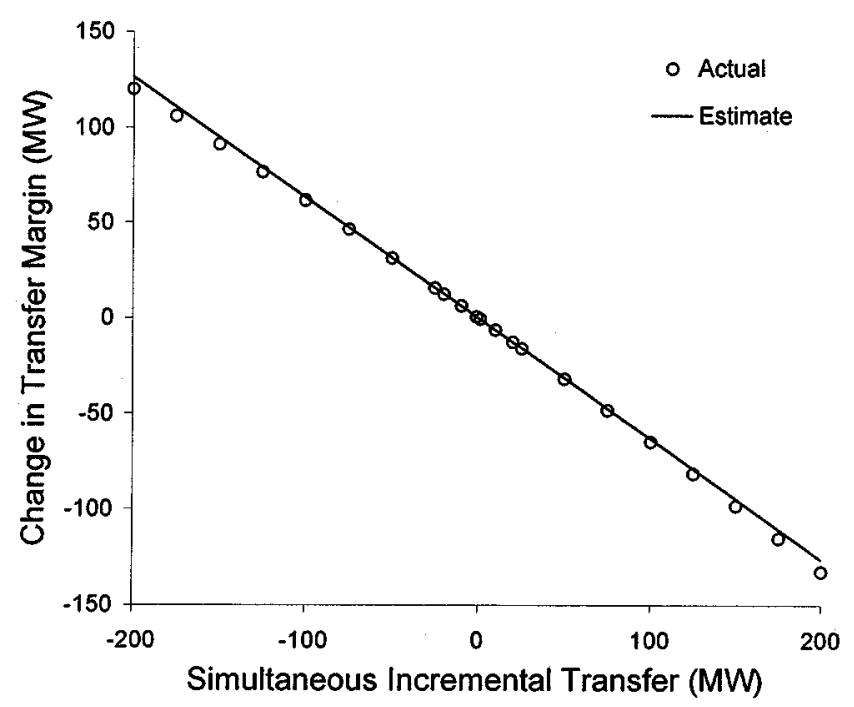

Fig. 4. Effect of simultaneous transfer on margin to critical VAR limit.

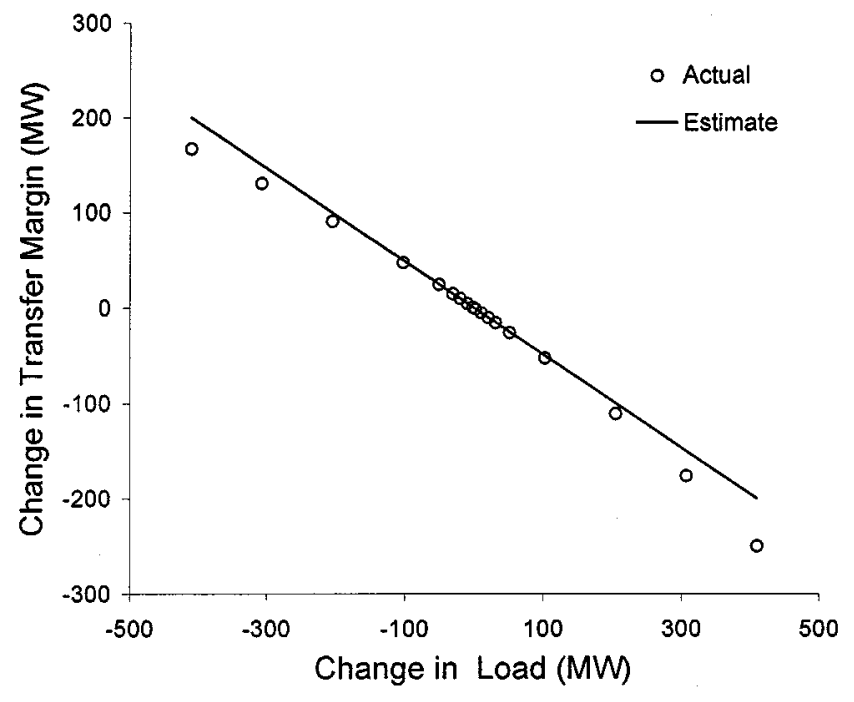

Fig. 5. Effect of Albany loading on margin to voltage limit.

All the results confirm the accuracy of the formula in predicting the transfer margin when small changes are made in a 


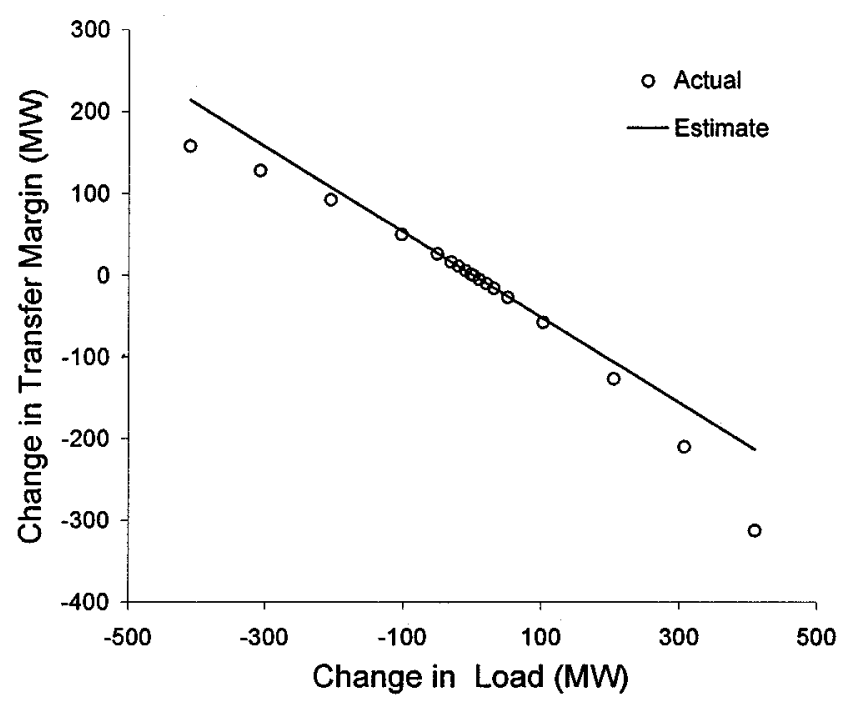

Fig. 6. Effect of Albany loading on margin to critical VAR limit.

parameter. For some parameters, the transfer margin is accurately predicted for large changes in the parameter. ${ }^{1}$ The range of parameter variation for which the prediction of transfer capability is accurate depends on the parameter considered, but generally is sufficiently large to support the usefulness of the first order approximation. Two possible sources of error in predicting the transfer margin for large parameter changes are the following.

- Nonlinearity: For fixed power system equations, the transfer margin varies nonlinearly with the parameter. For example, this is evident in the curvature of the actual results in Figs. 5 and 6.

- Structural Changes: As the parameter changes from its nominal value, the power system equations change when variables reach limits. After the equations change, the estimated changes in transfer margin computed with the equations valid at the nominal parameter value can be inaccurate. For example, this is evident in the sudden change in the actual results in Figs. 1 and 2 when a generator reactive power limit is encountered. It is clear that proximity of the transfer limited case to limits can in some cases limit the accuracy of the estimated changes in transfer margin. This proximity can be detected by the additional computation of state variable sensitivities suggested in Appendix A.

\section{HANDLing Multiple Limits}

A simple approach computes the sensitivity of the transfer margin to the single binding limit. In practice, particularly when the power system is uniformly and highly stressed, there are often other limits encountered just after the binding limit.

For example, Fig. 7(a) illustrates the next limit encountered at $N$ if the binding limit at $M$ is neglected. This next limit can be computed by running the continuation past the binding limit.

\footnotetext{
${ }^{1}$ We clarify meanings of "small" and "large." From a mathematical perspective, "small" means "infinitesimally small." From an engineering perspective, "small" can, for example, be $1 \mathrm{MW}$ for power variations and $0.1 \%$ for changes in voltage magnitude. Thus "small" corresponds to parameter changes for which the first order linearization will produce very accurate results. "Large" means not small.
}

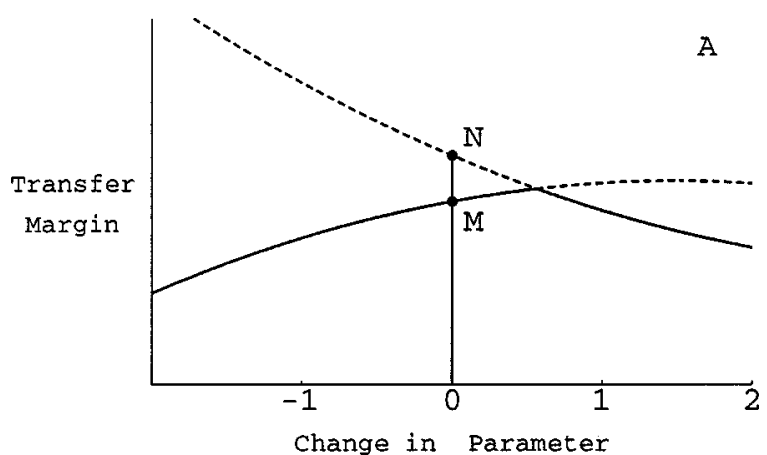

(a)

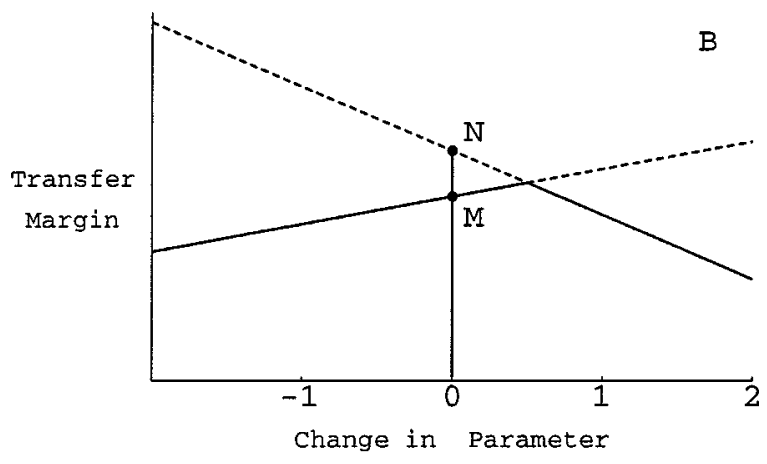

(b)

Fig. 7. Effect of parameter change on the next limit (dashed line) encountered just after the binding limit (solid line). (a) Actual limits. (b) Estimated limits.

Fig. 7(a) shows that if the parameter is increased past 0.56 , the next limit becomes the binding limit. In the situation of Fig. 7(a), the sensitivity of the transfer margin to both the binding limit and the next limit can be computed using the methods of this paper and the resulting linear estimates of the changes in these margins are illustrated in Fig. 7(b). For power system examples of this computation see [6].

Thus in the presence of multiple limits close to the binding limit, we recommend that the sensitivity of the corresponding transfer margins also be computed. Then the power system can be steered away from several security limits that may become binding. Finding the transfer margin sensitivity at each further limit requires recomputation of the transfer limited case. This is usually much quicker than the original computation of the binding transfer limited case, because if the further limit is relevant, it must occur soon after the binding limit. However, the re-computation of each transfer limited case is significantly more expensive than the sensitivity computation for each limit. Prediction of which voltage magnitude and line limits will occur soon after a binding limit can be done using the additional computation of state variable sensitivities suggested in Appendix A.

\section{RELATED WORK}

The primary tool used in industry for computing transfer capability margins is the dc loadflow model with power transfer distribution factors (PTDF) and outage transfer distribution factors (OTDF) computations (e.g., Power Technologies, Inc. program MUST [12]). It can be shown [7] that the sensitivity 
formula (3) reduces to PTDFs and OTDFs for the appropriate dc load flow models and this is illustrated in Appendix C. Thus this paper significantly generalizes standard sensitivity methods to encompass more accurate transfer capability calculations on more detailed models. In particular, account can be taken of power system nonlinearity, operator and automatic control actions, and voltage and reactive power limits. The detailed models also expand the range of parameters with respect to which transfer capabilities can be computed. For example, the sensitivity of a line flow limit to reactive power injection can be computed.

There is a close connection between continuation and optimization formulations for computing transfer capabilities. For example, continuation can be viewed as an interior point method of optimizing the amount of transfer. While there can be differences in the assumptions and accuracies of the various continuation and optimization methods of computing the transfer capability, the transfer capability sensitivity formula (3) is unaffected by these choices. The sensitivity formula (3) is derived using both continuation and optimization frameworks in Appendices $\mathrm{A}$ and $\mathrm{B}$, respectively.

Gravener and Nwankpa [10] have also nicely demonstrated the use of transfer margin sensitivities; the difference with this paper lies in the way the sensitivities are computed. In [10], the sensitivities are computed numerically by incrementing the parameter and rerunning whereas we suggest a fast analytical formula for the sensitivities.

The overall margin sensitivity approach which is generalized in this paper arose in the special case and restricted context of loading margins to voltage collapse caused by fold bifurcation [4], [8]. This paper considers transfer margins to general limits other than voltage collapse. The sensitivity of transfer margins to voltage collapse can be easily adapted from [8] and this material special to voltage collapse is not repeated here. The sensitivity formula of [8] differs from (3) in that $w$ stands for a different vector and that no event equation is used. ${ }^{2}$ Reference [11] demonstrates the use of the margin sensitivity methods of [8] for fast contingency screening for voltage collapse limits only. Testing of fast contingency screening using the more general security limits of this paper is future work.

The transfer capability sensitivity formula (3) was first stated in the workshop [9] and then in Greene [7]. This paper greatly extends the initial concepts in [9] by deriving the formula, testing it on a realistic power system, and assessing its practicality.

\section{CONCLUSION}

We show how the sensitivity of the transfer capability can be computed very quickly by evaluating an analytic formula at the binding limit. The sensitivities can be used to estimate the effect on the transfer capability of variations in parameters such as those describing other transfers, operating conditions or assumed data. The approach is consistent with current industrial practice using dc load models and significantly generalizes this practice to include more elaborate ac power system models and

${ }^{2}$ Greene [7] formulates an event equation for the fold bifurcation to obtain a formula of the form of (3) which does reduce to the formula of [8]. voltage and reactive power limits on power system operation. Once the transfer capability and corresponding binding limit and solved case have been computed, the first order sensitivity of this transfer capability to a wide range of parameters can be quickly computed. These first order sensitivities can contribute to the quick update of transfer capabilities when operating conditions or other transfers change. Moreover, the sensitivities can be used to select operator actions to increase transfer capability.

We conclude that after each computation of a transfer capability, it is so quick and easy to compute sensitivities of that transfer capability that this should be done routinely to extract the maximum amount of engineering value from each computation. In the case of predicting the effects of large parameter changes on transfer margins, even if more than first order accuracy is ultimately required, it is still desirable to first estimate the effects with first order sensitivities.

\section{APPENDIX}

\section{A. Derivation of Sensitivity}

Define

$$
H(x, \lambda, p)=\left(\begin{array}{c}
F(x, \lambda, p) \\
E(x, \lambda, p)
\end{array}\right)
$$

$H\left(x_{*}, \lambda_{0}+k t_{*}, p\right)=0$. Assume that $H$ is smooth and assume the generic transversality condition that

$$
\left.\left(H_{x} H_{\lambda} k\right)\right|_{*} \text { has rank } n+1 \text {. }
$$

Then the implicit function theorem implies that there are smooth functions $X(p), T(p)$ defined near $p_{*}$ with $X\left(p_{*}\right)=x_{*}$ and $T\left(p_{*}\right)=t_{*}$ such that

$$
H\left(X(p), \lambda_{0}+k T(p), p\right)=0 .
$$

Differentiating (7) yields

$$
\left.\left(H_{x} H_{\lambda} k\right)\right|_{*}\left(\begin{array}{c}
X_{p} \\
T_{p}
\end{array}\right)=-\left.H_{p}\right|_{*} .
$$

There is a nonzero row vector $w$ such that $\left.w H_{x}\right|_{*}=0 . w$ is unique up to a scalar multiple when $\left.H_{x}\right|_{*}$ has full rank, which is implied by (6). Premultiplying (8) by $w$ yields

$$
\left.w H_{\lambda} k\right|_{*} T_{p}=-\left.w H_{p}\right|_{*} .
$$

Condition (6) implies that $\left.w H_{\lambda} k\right|_{*}$ is not zero and hence (9) can be solved to obtain (3). The geometric interpretation of the quantities in (3) is that $\left(w H_{\lambda} k,-w H_{p}\right)$ is the normal vector to the hypersurface in $(t, p)$ space corresponding to the binding limit.

The sensitivity $X_{p}$ of the states at the binding limit is often useful and this can be obtained by solving (8). For example, $X_{p} \Delta p$ can be used to screen for cases where new limits would be violated (e.g., $x_{i}+X_{p} \Delta p[i] \geq x_{i}^{\max }$ ) [7].

\section{B. Derivation of Sensitivity in an Optimization Context}

An optimization formulation [16, ch. 7], [18] of the transfer margin determination is: Maximize the cost function $T=t$ subject to the equilibrium equations (1) and the limit equations 
$x_{i}=x_{i}^{\min }$ and $x_{i}=x_{i}^{\max }$ for all applicable $i$. This optimization can be solved to find the transfer-limited case equilibrium solution $\left(x_{*}, \lambda_{*}, p_{*}\right)$ and the binding limit (2). In order to use the notation of Appendix A, note that this solution is also the solution of the optimization: Maximize the cost function $T=t$ subject to

$$
\begin{aligned}
H\left(x, \lambda_{0}+k t, q\right) & =0 \\
p-q & =0 .
\end{aligned}
$$

To be able to quote a common optimization result in the sequel, it is convenient to introduce the parameters $p$ into (10) via the new variables $q$. The variables are now $X=(x, t, q)^{t}$. Write

$$
L=t-w H-v(p-q)
$$

where $w$ and $v$ are row vectors of Lagrange multipliers. Then, at the optimum solution, it is necessary that $0=\partial L / \partial X$, or, equivalently, that

$$
\begin{aligned}
w H_{x *} & =0 \\
1-\left.w H_{\lambda}\right|_{*} k & =0 \\
\left.w H_{p}\right|_{*}+v & =0 .
\end{aligned}
$$

Equation (13) is identical to (4), showing that the Lagrange multiplier $w$ must be proportional to the vector $w$ used in the rest of the paper [the length of the Lagrange multiplier $w$ is fixed by (14)]. It is well known in optimization theory (e.g., see [17] or, in the context of applications to minimum cost optimal power flow see [14]) that the sensitivity of the cost function to the constraints is given by the corresponding Lagrange multiplier. Thus $T_{p}=v$. Applying (15) and then (14) yields

$$
T_{p}=v=-\left.w H_{p}\right|_{*}=\frac{-\left.w H_{p}\right|_{*}}{\left.w H_{\lambda}\right|_{*} k}
$$

which is identical to the desired formula (3).

\section{C. dc Load Flow Example}

We show how the general formula (3) applies in a simple dc load flow example with six buses. The slack bus is numbered 0 . For the nonslack buses, write $\theta=\left(\theta_{1}, \theta_{2}, \theta_{3}, \theta_{4}, \theta_{5}\right)^{T}$ for the angles and $\lambda=\left(\lambda_{1}, \lambda_{2}, \lambda_{3}, \lambda_{4}, \lambda_{5}\right)^{T}$ for the power injections. The dc load flow equations are $F(\theta, \lambda)=X \lambda-\theta$. The transfer is from bus 3 to bus 4 so that $k=(0,0,1,-1,0)^{T}$. The limit on the transfer is overload on line 1-2 so that the limit equation is $E(\theta, \lambda)=b_{12}\left(\theta_{1}-\theta_{2}\right)-\lambda_{12} \max$. The parameter is $\lambda_{5}^{0}$, the base case power injection at bus 5. $F_{\theta}=-I$ and $E_{\theta}=$ $\left(b_{12},-b_{12}, 0,0,0\right)$ and hence $w=\left(b_{12},-b_{12}, 0,0,0,1\right)$. $F_{\lambda}=X$ and $E_{\lambda}=0 . F_{\lambda^{0}}=X(0,0,0,0,1)^{T}$ and $E_{\lambda^{0}}=0$. The transfer margin $T$ is the increase in transfer from bus 3 to bus 4 which causes the flow limit on line 1-2. Substitution in (3) gives the sensitivity of $T$ with respect to injection at bus 5

$$
T_{\lambda_{5}^{0}}=\frac{X_{15}-X_{25}}{X_{13}-X_{23}-X_{14}+X_{24}}=\frac{\rho_{12,5}}{\rho_{12,3}-\rho_{12,4}}
$$

where $\rho_{12, m}=b_{12}\left(X_{1 m}-X_{2 m}\right)$ is the well known sensitivity of the flow on line 1-2 with respect to power injection at bus $m$.

\section{REFERENCES}

[1] V. Ajjarapu and C. Christy, "The continuation power flow: A tool for steady state voltage stability analysis," IEEE Trans. Power Syst., vol. 7, pp. 416-423, Feb. 1992.

[2] C. A. Cañizares and F. L. Alvarado, "Point of collapse and continuation methods for large ac/dc systems," IEEE Trans. Power Syst., vol. 7, pp. 1-8, Feb. 1993.

[3] H.-D. Chiang, A. Flueck, K. S. Shah, and N. Balu, "CPFLOW: A practical tool for tracing power system steady-state stationary behavior due to load and generation variations," IEEE Trans. Power Syst., vol. 10, pp. 623-634, May 1995.

[4] I. Dobson and L. Lu, "Computing an optimum direction in control space to avoid saddle node bifurcation and voltage collapse in electric power systems," IEEE Trans. Automat. Contr., vol. 37, pp. 1616-1620, Oct. 1992.

[5] - "Voltage collapse precipitated by the immediate change in stability when generator reactive power limits are encountered," IEEE Trans. Circuits Syst., vol. 39, pp. 762-766, Sept. 1992.

[6] I. Dobson, S. Greene, R. Rajaraman, F. L. Alvarado, C. L. DeMarco, M. Glavic, A. DeSouza, R. Zimmerman, and R. J. Thomas. (2001) Transfer capability calculator and tutorial. . [Online]. Available: http://www.pserc.cornell.edu/tcc/.

[7] S. Greene, "Margin and sensitivity methods for security analysis of electric power systems," Ph.D. thesis, ECE Dept., Univ. Wisconsin, Madison, 1998.

[8] S. Greene, I. Dobson, and F. L. Alvarado, "Sensitivity of the loading margin to voltage collapse with respect to arbitrary parameters," IEEE Trans. Power Syst., vol. 12, pp. 262-272, Feb. 1997.

[9] S. Greene, I. Dobson, F. L. Alvarado, and P. W. Sauer, "Initial concepts for applying sensitivity to transfer capability," in Proc. NSF Workshop Available Transfer Capability, Urbana, IL, June 1997.

[10] M. H. Gravener and C. Nwankpa, "Available transfer capability and first order sensitivity," IEEE Trans. Power Syst., vol. 14, pp. 512-518, May 1999.

[11] S. Greene, I. Dobson, and F. L. Alvarado, "Contingency ranking for voltage collapse via sensitivities from a single nose curve," IEEE Trans. Power Syst., vol. 14, pp. 232-240, Feb. 1999.

[12] G. Heydt, Computer Analysis Methods for Power Systems. New York: Macmillan, 1987

[13] Transmission Transfer Capability Task Force, "Available transmission capability definitions and determination," North American Reliability Council, Princeton, NJ, June 1996.

[14] J. Peschon, D. S. Piercy, W. F. Tinney, and O. J. Tveit, "Sensitivity in power systems," IEEE Trans. Power Apparat. Syst., vol. PAS-87, pp. 1687-1696, Aug. 1968.

[15] P. W. Sauer and S. Grijalva, "Error analysis in electric power system available transfer capability computation," Decision Support Syst., vol. 24, pp. 321-330, 1999.

[16] T. Van Cutsem and C. Vournas, Voltage Stability of Electric Power Systems. Boston, MA: Kluwer, 1998.

[17] T. L. Vincent and W. J. Grantham, Optimality in Parametric Systems. New York: Wiley, 1981.

[18] T. Wu and R. Fischl, "An algorithm for detecting the contingencies which limit the inter-area megawatt transfer," in Proc. North Amer. Power Symp., Washington, DC, Oct. 1993, pp. 222-227.

Scott Greene (S'96-M'99) received the Ph.D. degree in electrical engineering from the University of Wisconsin, Madison, in 1998.

He is now a Senior Engineer with L. R. Christensen Associates, Madison. He is a registered professional engineer in Wisconsin.

Ian Dobson (M'89-SM'98) received the Ph.D. degree in electrical engineering from Cornell University, Ithaca, NY, in 1989.

He is now a Professor of Electrical Engineering at the Power Systems Engineering Research Center, ECE Department, University of Wisconsin, Madison. His interests are applications of bifurcations and nonlinear dynamics, electric power system stability, power electronics, and self-organized criticality.

Fernando L. Alvarado (S'67-M'67-SM'80-F'93) received the Ph.D. degree in electrical engineering from the University of Michigan, Ann Arbor, in 1972.

He is now a Professor in the Power Systems Engineering Research Center, ECE Department, University of Wisconsin, Madison. His main areas of interest are computer applications to power systems and large scale problems. 